\title{
Christelle Avril, Marie Cartier, Delphine Serre, Enquêter sur le travail. Concepts, méthodes, récits
}

\section{Sophie Bernard}

\section{(2) OpenEdition}

1 Journals

Édition électronique

URL : http://journals.openedition.org/travailemploi/5263

DOI : 10.4000/travailemploi.5263

ISSN : 1775-416X

Éditeur

DARES - Ministère du Travail

\section{Édition imprimée}

Date de publication : 15 juin 2011

Pagination : 71-72

ISSN : 0224-4365

\section{Référence électronique}

Sophie Bernard, «Christelle Avril, Marie Cartier, Delphine Serre, Enquêter sur le travail. Concepts, méthodes, récits », Travail et Emploi [En ligne], 126 | avril-juin 2011, mis en ligne le 24 janvier 2012, consulté le 22 septembre 2020. URL : http://journals.openedition.org/travailemploi/5263 ; DOI : https://doi.org/10.4000/travailemploi.5263

Ce document a été généré automatiquement le 22 septembre 2020.

(C) Direction de l'animation de la recherche, des études et des statistiques (Dares) 


\title{
Christelle Avril, Marie Cartier, Delphine Serre, Enquêter sur le travail. Concepts, méthodes, récits
}

\author{
Sophie Bernard
}

\section{RÉFÉRENCE}

Christelle Avril, Marie Cartier, Delphine Serre, Enquêter sur le travail. Concepts, méthodes, récits Paris, La Découverte, collection Grands repères, 2010

1 Si nombre d'ouvrages sont consacrés aux méthodes d'enquêtes sociologiques, Christelle Avril, Marie Cartier et Delphine Serre proposent dans celui-ci de se focaliser sur leur mise en œuvre dans un domaine spécifique : celui du travail. Pour autant, l'enjeu n'est pas seulement épistémologique. En rendant compte de l'étendue et de la richesse des connaissances accumulées depuis plus d'un demi-siècle par la sociologie du travail ainsi que des instruments dont elle s'est dotée, il s'agit pour les auteures de mettre au jour la spécificité de l'analyse sociologique dans un champ largement investi par d'autres "spécialistes ». La sociologie du travail offre en effet une "autre compréhension du travail » permettant d'éclairer les façons dont les travailleurs exercent et perçoivent leur activité professionnelle.

2 Pour en rendre compte, les auteures s'appuient de manière systématique sur des enquêtes empiriques présentées en détails et sélectionnées au préalable en fonction de la visibilité des méthodes employées ainsi que de leur accessibilité à un large public. Pour ces raisons, la priorité a été donnée à des enquêtes récentes permettant par là même de valoriser les travaux de jeunes chercheurs et de mettre en évidence le dynamisme de la recherche en sociologie du travail. Outre l'intérêt méthodologique de la démarche, il s'agit au travers de ces terrains menés dans des secteurs diversifiés et auprès de l'ensemble des PCS de mettre au jour les évolutions en cours dans le monde du travail. A partir de ces récits d'enquête, sont dégagées, dans une perspective 
s'inspirant des travaux d'Howard Becker et de "ses ficelles du métier", des pistes méthodologiques et théoriques pour l'étude du travail. En dévoilant les « coulisses » des enquêtes sociologiques, les auteures nous invitent ainsi à une « sociologie du travail en acte». Elles revendiquent par ailleurs «une sociologie du travail ouverte et décloisonnée " se traduisant par la référence à des traditions sociologiques très différentes, allant de la sociologie des organisations à la sociologie interactionniste américaine, en passant par la sociologie des professions et des groupes professionnels, la sociologie des relations professionnelles ou encore la sociologie de l'emploi. Cette ouverture se fait également jour au travers de l'articulation voulue avec d'autres domaines de la sociologie ou d'autres sciences sociales telles que l'histoire ou l'anthropologie.

3 L'ouvrage se compose de quatre chapitres. Le premier s'attache à la description des « situations de travail » au travers de trois objets : les dimensions officielles du travail, les pratiques informelles des travailleurs, le travail comme produit d'un système d'interactions. C'est en s'appuyant sur plusieurs enquêtes empiriques que les auteures dégagent ainsi des « pistes » pour décrire le travail « tel qu'il s'impose » mais aussi « tel qu'il est", incitant à croiser les méthodes (documents, observations, entretiens, statistiques) pour mieux comparer les dimensions officielles de l'activité et les pratiques au travail. Elles insistent par ailleurs sur la posture distanciée que doit adopter le sociologue afin de ne pas préjuger des frontières des situations de travail pour être à même de les appréhender dans leur globalité.

4 Le deuxième chapitre traite des "dispositions professionnelles en acte » entendues comme «des façons d'agir, de penser, de percevoir incorporées au cours de leur histoire et de leur socialisation professionnelle» (p. 73) par les travailleurs et qui se répercuteraient sur leurs pratiques et leur rapport au travail. Ces dispositions professionnelles se révèleraient au travers des variations entre groupes professionnels ou au sein d'un même groupe. Les auteures partent donc du postulat que les différentes façons d'exercer son travail et de le percevoir relèveraient de dispositions professionnelles distinctes. Si ces dernières façonnent indéniablement les pratiques professionnelles et le rapport au travail, elles n'en demeurent pas moins mêlées à d'autres éléments dont l'influence peine à être distinguée des premières. Face à cette difficulté, la méthodologie pour saisir les "dispositions professionnelles en acte" mériterait d'être davantage explicitée. En revanche, dans les " pistes » dégagées par les auteures, sont mises en évidence l'intérêt de la comparaison et sont fournis au lecteur des outils permettant de saisir le processus de socialisation professionnelle et ses effets. Le troisième chapitre prolonge le précédent puisqu'il s'agit d'y aborder la question des "positions et trajectoires sociales des travailleurs" au travers du concept de «trajectoire» de Pierre Bourdieu. Les auteures insistent ici sur l'importance de prendre en compte l'influence de l'histoire de vie des travailleurs ainsi que de leur vie hors travail sur leurs pratiques professionnelles et leur rapport au travail. Sont ainsi abordés les effets du sexe, de l'origine sociale, de l'appartenance générationnelle, mais également de l'origine ethnique et de la situation familiale et matrimoniale.

6 Enfin, le dernier chapitre aborde la question des conditions d'emploi et de leur influence sur le travail, et notamment sur les contraintes du travail, les relations entre travailleurs, l'action collective au travail, la socialisation professionnelle. Si l'emploi précaire - notamment au travers des cas du travail à temps partiel et de l'emploi intérimaire - est analysé, l'emploi «protecteur» n'est pour autant pas ignoré, la 
comparaison se révélant comme un outil pertinent pour prendre de la distance avec les catégories juridiques. Par ailleurs, sont mises en évidence certaines distinctions telles que celle entre rapport au travail et rapport à l'emploi ou encore entre précarité et instabilité. Les auteures insistent également tout particulièrement dans les "pistes " qu'elles tracent sur l'intérêt d'intégrer dans l'analyse l'ensemble des acteurs qui participent concrètement à la fabrique et à la mise en œuvre des règles juridiques.

7 Agréable à lire et organisé de manière claire et didactique, ce livre à destination pédagogique présente un intérêt indéniable pour les étudiants s'initiant à la sociologie du travail et s'imposera sans nul doute comme un ouvrage de référence en la matière. Pour autant, les chercheurs y trouveront également des pistes de réflexion susceptibles de nourrir leurs réflexions. Chacune des pistes méthodologiques et théoriques sont illustrées par des exemples concrets empruntés à des enquêtes de terrain choisies judicieusement. En dévoilant les « coulisses » de celles-ci, les auteures donnent ainsi à voir la sociologie en train de se faire et démontrent également la richesse de la comparaison et de l'articulation des méthodes pour analyser le travail. Les auteures revendiquent en effet une sociologie fondée empiriquement tout en insistant sur l'importance de lier méthode et théorie, en pensant cette dernière comme "des concepts en acte ». C'est peut-être là une des limites de l'ouvrage que ne pas renforcer davantage cette partie conceptuelle. Ainsi en va-t-il de la notion de travail qui mériterait à notre sens d'être précisée. Au centre de l'ouvrage, celle-ci se révèle pourtant problématique, raison pour laquelle il aurait peut-être été opportun de rendre compte des débats qu'elle suscite dans la discipline. De même, s'il est indiqué dès l'introduction qu'il ne s'agit pas de recenser de façon exhaustive l'ensemble des recherches se rattachant à la sociologie du travail, on peut néanmoins regretter que certains des questionnements classiques propres à la discipline ne soient pas évoqués (sur des thèmes tels que les compétences et qualifications, ou encore la rationalisation et la technique par exemple). En témoigne notamment l'absence dans la bibliographie des traités de sociologie du travail, pourtant représentatifs des débats ayant cours dans la discipline. La volonté louable d'ouverture et de décloisonnement revendiquée par les auteures - se traduisant par la référence à une pluralité de traditions sociologiques - se révèle particulièrement riche mais elle parait parfois se faire aux dépens de la sociologie du travail proprement dite. L'accent mis sur les dispositions professionnelles et sociales est en ce sens significatif. Si les prendre en compte est en effet indispensable pour toute enquête sociologique, la place occupée par celles-ci dans l'ouvrage questionne sur la perspective défendue par les auteures. S'agit-il réellement d'enquêter sur le travail ou celui-ci ne serait-il finalement qu'un prétexte pour étudier la stratification sociale et les inégalités? En dépit de l'ouverture revendiquée par les auteures, la sociologie du travail présentée ici ne s'inscrit-elle pas dans une orientation spécifique? Malgré ces quelques réserves et les questionnements suscités par l'ouvrage, au final, le pari semble gagné. Au travers de cette « sociologie en acte », cet ouvrage devrait sans nul doute insuffler aux étudiants le goût de la sociologie et du travail de terrain. 


\section{AUTEURS}

\section{SOPHIE BERNARD}

Maître de conférences en sociologie, IRISSO, université Paris Dauphine 